\title{
Transforming optics: an interview with Sir John Pendry
}

Advanced Photonics spoke with Sir John Pendry of Imperial College London, United Kingdom, about the history of transformation optics.

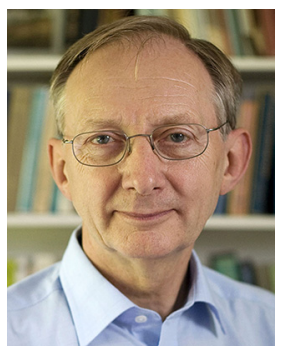

Sir John Pendry
Advanced Photonics: How did you come up with the idea of transformation optics? Pendry: In the mid 1990s I was working on finite element codes to study photonic crystals. These were coded in a Cartesian coordinate system which corresponded well with the photonic crystal geometry. Then we wanted to look at photonic crystal fibers where the obvious coordinate system was a cylindrical one. Being a lazy person I asked if there was some way I could avoid completely rewriting the codes and then realized that I could use the Cartesian/cylindrical transformation to find effective values of epsilon and mu in the Cartesian system which made it behave like a cylindrical one, and the old codes worked like a dream for the fibers. Along with the algebraic formulae was the realization that field lines were also conserved under the transformation and merely rearranged in space behaving as if they were glued to the distorting coordinate frame. This provides a valuable physical insight into the transformation process which goes far beyond the algebraic formulae. Subsequently in 2003 David Smith and I used the transformation optics trick to re-engineer the perfect lens into a perfect magnifying glass. This work was part of a confidential contract and although we patented the concept we did not publish.

Advanced Photonics: What was the optics community response to this idea?

Pendry: The response to our original 1996 paper was decidedly muted: it still has only 343 citations most of them in the cloaking context. The idea only really burst into prominence after a DARPA meeting in April 2005 on negative index material held in San Antonio. Val Browning, the organizer, suggested that I should 'ginger things up' so, it being a military meeting, I decide to show how to make something invisible. My reference to Harry Potter appealed to Val who was the mother of several young children. It was intended as a joke, being only one of very many applications of transformation optics but has subsequently taken on a life of its own.

Advanced Photonics: How long did it take from the idea to the first published article? Did you have any problems with convincing editors and/or referees?

Pendry: After the San Antonio talk David Smith and his team almost immediately started work on an experimental realization, succeeding only a few months after San Antonio, and leading to a couple of publications a year later. One was on the theory, the other on the experimental realization. This was a complicated process as our institutions were focused on patent rights, which had to be sorted. The referees were somewhat sceptical of the first paper but were eventually persuaded to accept it. The press interest was absolutely astonishing and this was the first time that my science had entered the popular domain in any serious way. Quite apart from its scientific significance, transformation optics through the cloak has proved a valuable vehicle for outreach to a diverse community.

Advanced Photonics: Is transformation optics possible to realize only with metamaterials?

Pendry: Metamaterials make it easy to implement transformation optics, which often produce rather demanding material parameters hard to realize in natural materials. They certainly were key to the first cloak and helped spread the enthusiasm for cloak-making very widely in the community. But there have been realizations of the technology at optical frequencies using naturally anisotropic materials such as calcite. Shuang Zhang's group in Birmingham and Baile Zhang in Singapore both produce versions of this type of cloak.

Advanced Photonics: What advances did the transformation optics approach provide in nanophotonics and metamaterials, which would not be possible otherwise?

Pendry: As the review [in this issue] attests there have been many applications of transformation optics. My own personal interests have of late focused on how structure radically affects surface plasmons. These surface electron density waves have wavelengths much shorter than the free space wavelength of light and are candidates for subwavelength (a)

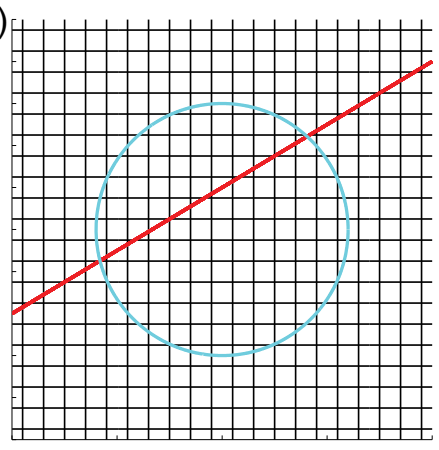

(b)

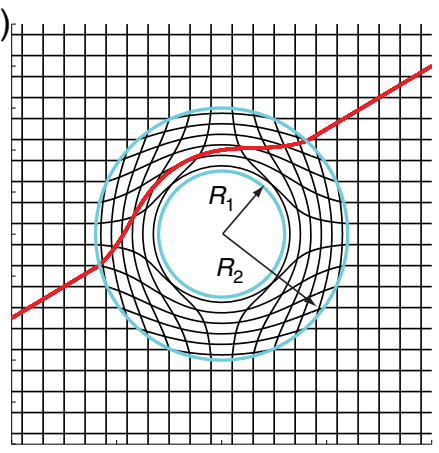

(c)

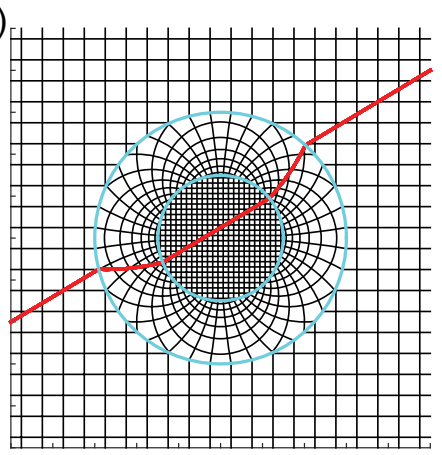

The electromagnetic invisibility cloak is an example of transformation optics achievements [from Fig. 2 in J. Zhang, J. B. Pendry, and Y. Luo, "Transformation optics from macroscopic to nanoscale regimes," Adv. Photonics 1, 014001 (2019)].

(C) 2019 SPIE and CLP. [DOI: 10.1117/1.AP.1.1.010502] 
control of radiation. Transformation optics is a tool ideally suited to understanding and designing novel subwavelength structures-metasurfaces if you like - that control and shape the surface plasmons. It gives both intuitive understanding and at the same time is precise at the level of Maxwell's equations.

Advanced Photonics: Is the transformation optics approach specific to electromagnetic waves or can it be exploited in other areas of physics? Pendry: It has been fascinating to see the concept spread into many other areas of wave physics, particularly into acoustics, which I am sure will be one of the many areas where practical applications will be found. Sebastien Guenneau has even made a proposal that strategic buildings could be cloaked from earthquakes!

Advanced Photonics: What practical applications did transformation optics spin off?
Pendry: As always with a new technology the first practical applications are to relatively mundane problems. So far, people have solved such problems as how to make a sharp bend in a wave guide without any back reflection. This problem is easily and almost trivially solved by transformation optics. There have been applications to 'spoofing,' i.e., making an object to be seen as a quite different object on a radar screen. In the optical domain, the technique can reveal many different ways of concentrating light into a nanoscale focus with applications to molecular sensing and to low-power nonlinearity.

Advanced Photonics: What will be future of transformation optics? Pendry: The technology is well on its way to being embedded as a standard design technique for the control of radiation, as well as providing physical insight where waves interact with complex structures. 